\title{
Microsurgical options after the failure of left colon interposition graft in esophagogastric reconstruction
}

\author{
Han Gyu Cha, \\ Hyung Hwa Jeong, \\ Eun Key Kim \\ Department of Plastic Surgery, Asan \\ Medical Center, University of Ulsan \\ College of Medicine, Seoul, Korea
}

\begin{abstract}
Colon interposition is commonly used for esophageal reconstruction in patients with a previous gastrectomy. However, when colon interposition fails and alternative reconstruction is required, there are few options for reconstructing the long segment from the esophagus to the stomach. Here, we report on cases of esophagogastric reconstruction with limited alternative options after the failure of transverse and left colon interposition. In these cases, reconstruction was performed using two different microvascular methods: double-pedicle jejunal free flap and supercharged ileocolic interposition graft.
\end{abstract}

Keywords: Colon / Esophageal neoplasms / Jejunum / Microsurgery

\section{INTRODUCTION}

Currently, gastric tube or gastric pull-up are the first-line options as esophageal substitutes for reconstruction after esophagectomy in patients with esophageal cancer $[1,2]$. However, in cases in which the stomach is not available owing to prior or simultaneous gastrectomy for various reasons, using the colon or jejunum are the next choices [3]. Traditionally, colon interposition has been the most widely used conduit procedure, with advantages of long length, less reflux, and reservoir-like capacity [2]. The transverse and left colon is frequently used as a conduit owing to its adequate length and reliable blood supply compared with the right colon. This is mainly because of the constant existence of collateral networks between the middle colic and left colic arteries, whereas collateral circulation between the middle colic and right colic arteries is not constantly present [4]. In addition, the left colon has a thicker wall and a smaller diam-

\section{Correspondence: Eun Key Kim}

Department of Plastic Surgery, Asan Medical Center, University of Ulsan College

of Medicine, 88 Olympic-ro 43-gil, Songpa-gu, Seoul 05505, Korea

E-mail: nicekek@korea.com

Received December 12, 2018 / Revised December 29, 2018 / Accepted January 3, 2019 eter than the right colon [5].

However, when failure of transverse and left colon interposition occurs, alternative options are needed for reconstructing the long segment from the esophagus to the stomach. Here, we report cases of esophagogastric reconstruction with limited alternative options after the failure of transverse and left colon interposition, for which two different microvascular methods were used: double-pedicle jejunal free flap and supercharged ileocolic interposition.

\section{CASE REPORTS}

\section{Case 1}

A 65-year-old male patient with squamous cell carcinoma of the upper esophagus underwent robot-assisted esophagectomy and gastrectomy followed by reconstruction with a transverse and left colon interposition graft based on the middle colic artery as a conduit. However, anastomosis site leakage due to subtotal conduit necrosis was detected after 10 days, and cervical esophagostomy needed to be done. To salvage the remnant colon conduit, jejunal free flap reconstruction was planned. Be- 
cause of the need for a long jejunum length (about $60 \mathrm{~cm}$ ), the left inferior mesenteric vessels in the proximal portion and the right inferior mesenteric vessels in the distal portion were harvested together to perform double-pedicle flap surgery. Microvascular anastomosis was done retrosternally between the left inferior mesenteric vessels and the left internal mammary vessels, and between the right inferior mesenteric vessels and the right internal mammary vessels. The proximal jejunum was anastomosed to the remnant esophagus in an end-to-end fashion, and the distal jejunum was anastomosed to the remnant colon conduit in a side-to-side fashion. Perfusion was confirmed by checking for constant peristalsis of the jejunum (Fig. 1). There was no sign of fistula or leakage at the anastomotic site at 3 weeks postoperatively, and oral feeding was started (Fig. 2).

\section{Case 2}

A 64-year-old patient presented with squamous cell carcinoma of the mid portion of the esophagus. There was no sign of distant metastasis except for suspected metastasis at the paratracheal lymph nodes. Total esophagectomy and gastrectomy were done followed by transverse-sigmoid colon interposition from
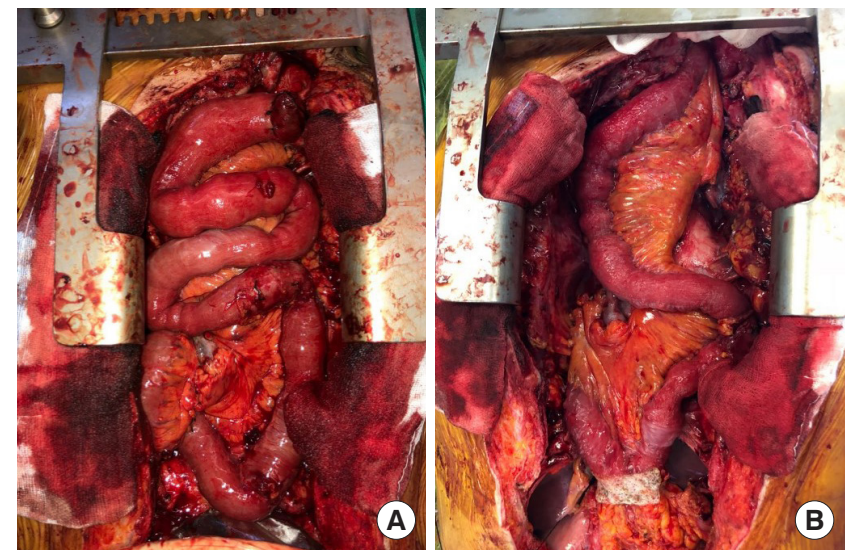

Fig. 1. Overview photographs. (A) Photograph taken immediately after anastomosis of double-pedicle jejunal free flap. (B) Photograph taken 2 hours after anastomosis showing the peristalsis of jejunum.
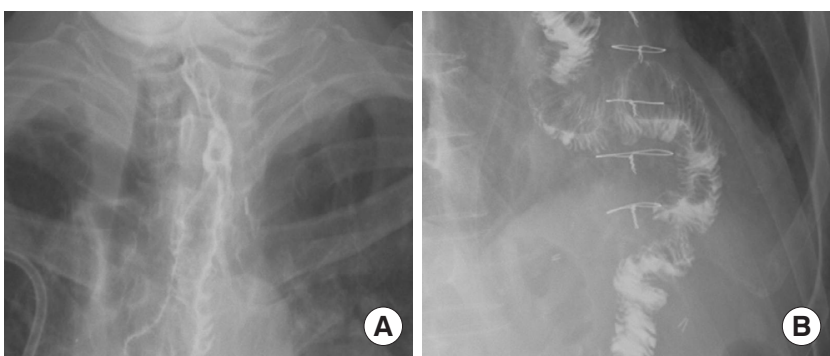

Fig. 2. Esophagography images of the 65-year-old patient (case 1) taken 3 weeks after surgery. No leakage or fistula was seen. (A) Distal portion. (B) Proximal portion. the esophagus to the jejunum with colon conduit formation based on the middle colic artery. However, the conduit was removed and feeding jejunostomy was performed because of ischemic necrosis of the conduit 2 weeks after the primary surgery. To resume oral feeding, revision with alimentary tract interposition was required, and the total length of the defect to be reconstructed was $40 \mathrm{~cm}$.

There was severe adhesion at the previous jejunostomy area extending to the spleen and liver, which precluded the use of the jejunum. Owing to the previous use of the transverse-descending colon with the left and middle colic arteries as a conduit, the right ascending colon and ileum were the only choices. The remnant right colon and distal ileum, with a total length of $50 \mathrm{~cm}$, along with the right colic artery as the pedicle were harvested to cover the esophageal defect. The ileocolic artery was dissected and clipped for high anastomosis, and the ileal portion was transposed to the resection stump of the esophagus in retrosternal placement. Because the long segment of the ileum was included in the graft to be interposed, supercharging of the ileocolic vessel was mandatory to guarantee full blood supply to the overall graft. The left internal mammary artery and vein were prepared in retrosternal space and anastomosed with the ileocolic vessels in an end-to-end fashion (Fig. 3). The perfusion of the interposed intestine was confirmed by checking for fresh bleeding from the serosa and constant peristalsis of the graft. Ileoesophageal anastomosis was completed in an end-toside fashion, and the distal end of the interposed ascending colon was anastomosed to the jejunum in an end-to-side fashion. Finally, the proximal ileal stump was anastomosed to the sigmoid colon stump (Fig. 4). The overall scheme of the procedure is delineated in Fig. 4. There was no sign of fistula or leakage at the anastomotic site at 3 weeks postoperatively, and oral feeding was started (Fig. 5).

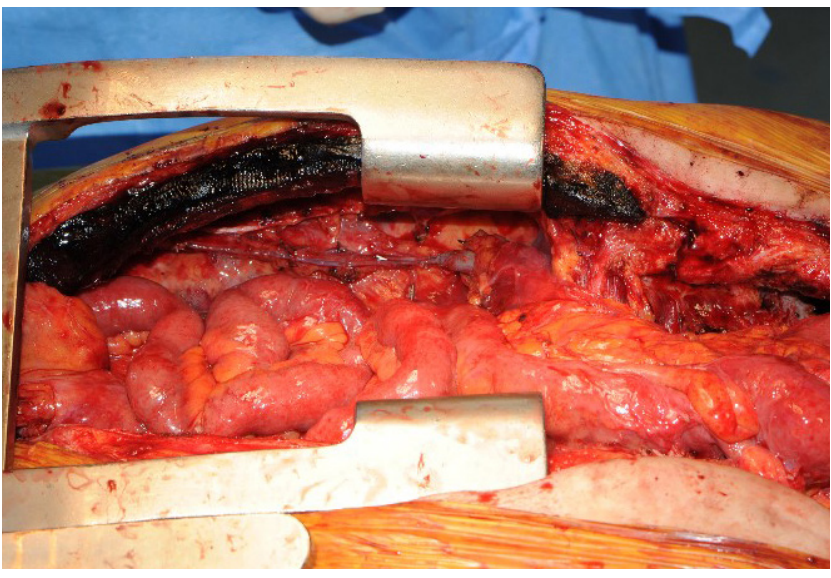

Fig. 3. Supercharged ileocolic artery and vein. The ileocolic vessels were anastomosed to the left internal mammary artery and vein. 


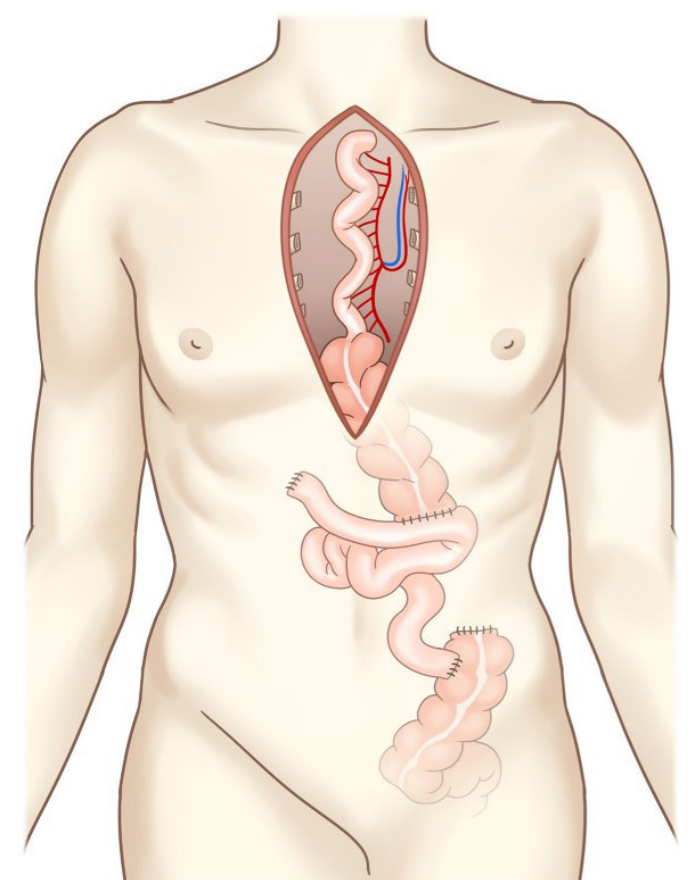

Fig. 4. Supercharged ileocolic interposition procedure (overall scheme).

\section{DISCUSSION}

The use of the colon in esophageal reconstruction was first introduced in 1911, and the colon remains the first-line alternative to the gastric tube. As long segments are needed to substitute the entire length of the esophagus and stomach in total esophagectomy and gastrectomy, the colon has an advantage over the jejunum in terms of its longer length. Most surgeons prefer to use a left or extended left colon interposition graft based on the ascending marginal branch of inferior mesenteric vessels, owing to its reliable vascular arcade. However, the variation of mesenteric vessels around the colon may cause unpredictable ischemia. Although preoperative angiography provides information on the status of the mesenteric blood supply, twisting of the colon because of its position after cervical anastomosis and invisible monitoring may lead to inevitable necrosis. According to previous studies, the graft loss rate in left colon interposition was from $3.8 \%$ to $9.3 \%$ [6-8]. Furthermore, the incidence of anastomotic leaks and graft ischemia was higher if a long segment of the colon was used rather than short-segment grafting [9].

In cases of colon interposition failure, the free jejunal graft can be used as a solution. The jejunum itself has advantages over the colon as an esophageal conduit with easy mobilization, similar diameter to the esophagus, and active peristalsis. Thus, some surgeons prefer pedicled jejunum as the first option in

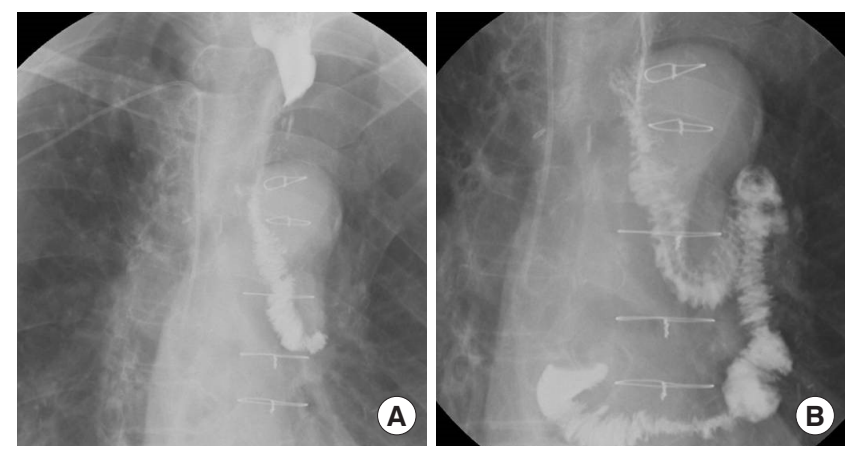

Fig. 5. Esophagography images of the 64-year-old patient (case 2) taken 3 weeks after surgery. No leakage or fistula was seen. (A) Distal portion. (B) Proximal portion.

esophageal reconstruction. However, because a tight arrangement of the mesenteric arcade may result in vascular compromise to the distal segment, it has limitations for short-segment use and free jejunal graft is often used by microsurgeons. In free jejunal graft, a single vascular pedicle generally vascularizes 25 $30 \mathrm{~cm}$ of the jejunum, and esophageal reconstruction with the double-pedicle jejunal free flap has been reported to be able to transfer up to $60 \mathrm{~cm}$ of free jejunum [10]. Similarly, in our case, we obtained a $60 \mathrm{~cm}$ length of the jejunum with two pedicles in the distal and proximal portions. This double-pedicle free jejunum graft has advantages not only in terms of its vascular supply but also in its easy arrangement. Especially when using both internal mammary vessels retrosternally, it is easy to position the jejunum freely before anastomosis and perform microanastomosis in a wide surgical field.

However, if the jejunum is unavailable like in our first case, the only option for a long-segment esophageal conduit is ileocolic interposition. Ileocolic interposition is one of the variant techniques of right colon interposition including the ascendingtransverse, ileum-transverse colon, and ascending colon alone. Generally, the ileocolic interposition graft is based on the middle colic vessels, and many studies using this technique have been published. Moreover, the middle colic vessel was used exclusively as a pedicle in cases of supercharged ileocolic interposition graft augmenting blood flow with the ileocolic artery [1113]. Different from previous studies, we have used the right colic artery as a pedicle, which was the only available vessel to feed the ascending colon. To secure sufficient length of the ileum as a conduit, the terminal ileum arcade and the communicating arcade between the ileal branch of the ileocecal artery and the marginal artery were preserved meticulously before transecting the ileum. This manner of using the right colic artery as a pedicle is not always available because the right colic artery is reported to be absent in $12.6 \%$ of patients and only $68 \%$ of patients have 3 separate vessels including the middle colic, right 
colic, and ileocolic arteries [14]. Thus, preoperative angiography and intraoperative Doppler pulse flowmetry should be performed to confirm the vascular anatomy along the superior mesenteric artery and its branches.

In selecting recipient vessels for vascular augmentation, we prefer the internal mammary vessels with reliable constancy. In patients with failed primary surgery, vessels in the head and neck region, such as the transverse cervical, superior thyroid, lingual, and facial arteries, are often adhered to the surrounding scar tissue after multiple surgeries or radiation therapy. Moreover, for anastomosis to these vessels, the subcutaneous route is required and this may result in disfigurement (subcutaneous bulging) as well as the risk of compression of the graft, which might compromise graft perfusion. As a retrosternal approach is required in most cases owing to manipulation of the previously operated conduit by the thoracic surgeon, it is considerably easy to approach both sides of the internal mammary vessels simultaneously in case of a double-pedicle jejunal free flap. Thus, we suggest the internal mammary vessel as the most reliable vessel for vascular supercharging, as in breast reconstruction.

Long-segment esophagogastric reconstruction remains a surgical challenge. The single most important factor in long-segment esophagogastric reconstruction is sufficient blood supply to the graft segment. As there are a few options after the failure of colon interposition in esophagogastric reconstruction, the thoracic surgeon, general surgeon, and microsurgeon should all be aware of the limited options and should be prepared to perform microsurgical techniques. As additional vascular supplement is essential in the long-segment jejunum or ileum-colon, understanding of the anatomy along the mesenteric arcade should is required. Selection of adequate recipient vessels is another important prerequisite in completing microsurgical vascular augmentation.

In conclusion, in cases of failed colon interposition in esophagogastric reconstruction, the double-pedicle free jejunal graft should be considered as a first-line option and supercharged ileocecal interposition can be an alternative option where the jejunum cannot be utilized. The microsurgical approach is essential in secondary long-segment esophagogastric reconstruction, and phased cooperation among the thoracic surgeon, general surgeon, and microsurgeon is needed along with a thorough understanding of the vascular anatomy of the gastrointestinal tract.

\section{NOTES}

\section{Conflict of interest}

No potential conflict of interest relevant to this article was reported.

\section{Ethical approval}

The study was performed in accordance with the principles of the Declaration of Helsinki. Written informed consents were obtained.

\section{Patient consent}

The patients provided written informed consent for the publication and the use of their images.

\section{ORCID}

$\begin{array}{ll}\text { Han Gyu Cha } & \text { https://orcid.org/0000-0003-3059-1334 } \\ \text { Hyung Hwa Jeong } & \text { https://orcid.org/0000-0002-9981-6506 } \\ \text { Eun Key Kim } & \text { https://orcid.org/0000-0002-3986-6886 }\end{array}$

\section{REFERENCES}

1. Luan A, Hunter CL, Crowe CS, Lee GK. Comparison of outcomes of total esophageal reconstruction with supercharged jejunal flap, colonic interposition, and gastric pull-up. Ann Plast Surg 2018;80(5S Suppl 5):S274-8.

2. Watanabe M, Mine S, Nishida K, Kurogochi T, Okamura A, Imamura Y. Reconstruction after esophagectomy for esophageal cancer patients with a history of gastrectomy. Gen Thorac Cardiovasc Surg 2016;64:457-63.

3. Chen HC, Tang YB. Microsurgical reconstruction of the esophagus. Semin Surg Oncol 2000;19:235-45.

4. Bothereau H, Munoz-Bongrand N, Lambert B, Montemagno S, Cattan P, Sarfati E. Esophageal reconstruction after caustic injury: is there still a place for right coloplasty? Am J Surg 2007; 193:660-4.

5. Wormuth JK, Heitmiller RF. Esophageal conduit necrosis. Thorac Surg Clin 2006;16:11-22.

6. Furst H, Huttl TP, Lohe F, Schildberg FW. German experience with colon interposition grafting as an esophageal substitute. Dis Esophagus 2001;14:131-4.

7. Hagen JA, DeMeester SR, Peters JH, Chandrasoma P, DeMeester TR. Curative resection for esophageal adenocarcinoma: analysis of 100 en bloc esophagectomies. Ann Surg 2001; 234:520-30.

8. Klink CD, Binnebosel M, Schneider M, Ophoff K, Schumpelick $\mathrm{V}$, Jansen M. Operative outcome of colon interposition in the treatment of esophageal cancer: a 20 -year experience. Surgery 2010;147:491-6.

9. Cerfolio RJ, Allen MS, Deschamps C, Trastek VF, Pairolero PC. Esophageal replacement by colon interposition. Ann Thorac Surg 1995;59:1382-4.

10. Germain MA, Hartl DM, Boutin P, Marandas P, Luboinski B. Total esophagoplasty using a doubly vascularized free jejunal 
transplant: a last resort in two patients. Plast Reconstr Surg 2003;111:801-4.

11. Doki Y, Okada K, Miyata H, Yamasaki M, Fujiwara Y, Takiguchi S, et al. Long-term and short-term evaluation of esophageal reconstruction using the colon or the jejunum in esophageal cancer patients after gastrectomy. Dis Esophagus 2008;21:1328.

12. Kesler KA, Pillai ST, Birdas TJ, Rieger KM, Okereke IC, Ceppa $\mathrm{D}$, et al. "Supercharged" isoperistaltic colon interposition for long-segment esophageal reconstruction. Ann Thorac Surg 2013;95:1162-8.

13. Saeki H, Morita M, Harada N, Egashira A, Oki E, Uchiyama H, et al. Esophageal replacement by colon interposition with microvascular surgery for patients with thoracic esophageal cancer: the utility of superdrainage. Dis Esophagus 2013;26:50-6.

14. Peters JH, Kronson JW, Katz M, DeMeester TR. Arterial anatomic considerations in colon interposition for esophageal replacement. Arch Surg 1995;130:858-62. 